\title{
Inhibition of FAK prevents blister formation in the neonatal mouse model of pemphigus vulgaris
}

\author{
María P. Gil ${ }^{1}$, Teresa Modol$^{2}$, Agustín España ${ }^{1}$ and María J. López-Zabalza ${ }^{2}$ \\ ${ }^{1}$ Department of Dermatology, School of Medicine, University Clinic of Navarra, \\ University of Navarra, Navarra, Spain; ${ }^{2}$ Department of Biochemistry, School of \\ Medicine, University Clinic of Navarra, University of Navarra, Navarra, Spain
}

Correspondence: Agustín España, Department of Dermatology, School of Medicine, University Clinic of Navarra, University of Navarra, PO Box 4209, Pamplona 31080, Navarra, Spain, Tel.: 34-948-255400, Fax: 34-948-296500, email: aespana@unav.es

\begin{abstract}
Pemphigus vulgaris (PV) is an autoimmune blistering skin disease characterized by suprabasal acantholysis and by autoantibodies against desmoglein 3 localized on desmosomes. In addition, caspases also seem to participate in this blistering disease. Focal adhesion kinase (FAK) is a non-receptor tyrosine kinase involved in cytoskeleton remodelling and formation and disassembly of cell adhesion structures. We have previously demonstrated that HER (human epidermal growth factor receptor related) isoforms, Src (Rous sarcoma) and mammalian target of rapamycin (mTOR), three molecules implicated in signalling processes, take part in suprabasal acantholysis and apoptosis induced by PV-IgG in a mouse model. Our aim was to investigate whether upregulation of FAK is implicated in the development of PV lesions. Herein, using a mouse model, PV-IgG administration showed an increased level of FAK phosphorylated on 397 and 925 tyrosine residues in the basal layer of epidermis.

When mice were pretreated with a FAK inhibitor (FI), the acantholysis of the basal layer of epidermis was absent. More interestingly, we observed that phosphorylated FAK (Y397925) decreased when HER isoforms, Src, mTOR and pan-caspases inhibitors were employed before PV-IgG administration. In addition, pretreatment with the FI before PV-IgG injection prevented the changes in both Bax and Bcl-2 expression and caspase- 9 and caspase- 3 activities induced by PV-IgG. Finally, FI reduced the expression of phosphorylated Src and mTOR in the basal cells of epidermis. In conclusion, our data reveal a novel role of phosphorylated FAK (Y397/925) in PV development involving HER isoforms, Src and mTOR kinases.
\end{abstract}

KEY WORDS: acantholysis - caspases - focal adhesion kinase - mouse model pemphigus vulgaris 


\section{INTRODUCTION}

Pemphigus vulgaris (PV) is an autoimmune blistering skin disease characterized by suprabasal detachment of epidermal cells known as acantholysis and by anti-epidermal autoantibodies $(1,2)$ against the extracellular domain of desmoglein (Dsg) 3 with or without anti-Dsg1 (3). There are other non-desmoglein autoantibodies detected in the sera of patients with PV (4,5), such as acetylcholine receptors (6), E-cadherin (7) or antimitochondrial autoantibodies (8), which have also been implicated in the acantholytic process.

The mechanisms by which these autoantibodies induce acantholysis in PV may be due to a direct effect by binding to their target and may be mediated by steric hindrance andor by triggering signal transduction pathways in the keratinocyte (9). In this sense, several intracellular signalling molecules are triggered in PV after binding of autoantibodies to keratinocytes (10-16). Additionally, apoptosis has been suggested to play a role in PV (17-22). It is interesting to observe that both apoptosis and acantholysis are inhibited when inhibitors for specific intracellular signalling pathways are used (16). These observations have led to the concept of apoptolysis (23). In addition, it has been established that although apoptosis appears to be a late event and may not be indispensable for acantholysis in PV, activation of several members of the caspase family takes place in early PV lesions triggering the blistering response $(22,24)$.

Recently, adhesion-linked kinases, such as focal adhesion kinase (FAK), that regulate several cellular functions have been receiving increasing attention. FAK is a soluble non-receptor tyrosine kinase expressed by almost all tissues and cell types involved in cytoskeleton remodelling and in the formation and disassembly of cell adhesion structures $(25,26)$. Its presence in mouse epidermis and hair follicle has been detected in a pattern that was primarily plasma membrane associated (27). There are several tyrosines that could be phosphorylated in FAK, including Y397, Y407, Y576, Y577, Y861 and Y925. The residue 397 is the only tyrosine known to be autophosphorylated, whereas the others appear to be phosphorylated by Src (28). Although FAK has been implicated in promoting an invasive phenotype in some human tumors, it is not yet known whether this kinase plays an active role in PV lesions as well. Interestingly, Penneys found that FAK protein was mainly overexpressed in acantholytic cells in PV and pemphigus foliaceus lesions, but only scarce staining was noted in acantholytic cells in Hailey-Hailey disease, and it could not be detected in keratinocytes in spongiotic or dyskeratotic processes (29).

More recently, Chernyavsky et al. (13) have demonstrated that stimulation of a9 acetylcholine receptor (a9AchR) upregulates the phosphorylation of adhesion molecules of the focal adhesions (including FAK) and intercellular junctions (including Dsg3) as well as cytokeratins. Inhibition of a9AchR interferes with the phosphorylation of adhesion and cytoskeletal proteins and also disrupts cell-cell cohesion. In addition, Nguyen et al. (30) described the presence of circulating autoantibodies against a9AchR in patients with PV regulating keratinocyte adhesion.

Currently, in vivo experiments using the pan-HER isoform inhibitors CL-387,785 (CL) (31), the Src inhibitor PP1 (32), the mammalian target of rapamycin (mTOR) inhibitor rapamycin (RP) (33), the pan-caspases inhibitor cpm-VAD-CHO (PCI) (34) and the FAK inhibitor PF-573,228 (FI) (35) have been successfully employed to explore the involvement of these signalling molecules in several inflammatory disorders. 
Our group has already demonstrated a relevant role of HER isoforms, Src, mTOR (16) and neural nitric oxide synthase (nNOS) (15) activities in PV lesions by showing that specific inhibitors of these molecules are capable of reducing blister formation. Additionally, it has also been reported that rapamycin may inhibit IGF-I-induced Factin reorganization and phosphorylation of the focal adhesion proteins by the disruption of mTOR- raptor complex (36). Therefore, it is tempting to speculate on the role of FAK in the detachment of epidermal cells in PV.

Based on these findings and our previous results, we investigated the role of FAK in acantholysis in PV lesions in a mouse model. Herein, we observed for the first time that activated FAK takes part in PV acantholysis in a process mediated by changes in both Bax and Bcl-2 levels and caspase- 9 and caspase- 3 activities. We describe a novel role of this kinase (FAK) involving HER isoforms, Src and mTOR kinases in PV lesions.

\section{MATERIAL AND METHODS}

\section{Human sera}

Our study is based on experiments using IgG fractions isolated from the sera of three patients with active mucocutaneous PV and the serum of a healthy donor (NHS). Each of these three patients gave their signed consent to use their sera for experimental studies. The diagnosis of PV was based on criteria established elsewhere (37). By indirect immunofluorescence (IF) using monkey oesophagus, we found that the titre of intercellular antibodies for PV sera ranged from $1 / 320$ to $1 / 640$. Immunological characterization of PV and NHS sera was included to study the circulating antibodies against Dsg1 and Dsg3 using a commercially available enzyme-linked immunosorbent assay ELISA kit, following the manufacturer's recommendations (MBL Naka-Ku, Nagoya, Japan). The index value for Dsg1 antibodies for PV sera ranged from 60 to 189 , and that for Dsg3 antibodies from 77 to 173 . IgG fractions from the patients with PV and from NHS group were prepared as previously described $(15,16)$.

\section{Testing IgG pathogenicity using the passive transfer animal model}

All of our experiments were conducted using a mouse model of passive transfer of IgG (12). C57BL/6J mice were obtained from the Jackson Lab (Bar Harbor, ME, USA). The study was performed on 24- to 48-h-old neonatal mice (weighing 1.5-2.0 g) from a colony maintained at the Centre of Animal Control at the University of Navarra. IgG from all three PV and NHS sera were injected intradermally into neonatal mice (2 $\mathrm{mg} / \mathrm{g}$ ) ( $\mathrm{n}=3$ for each $\mathrm{IgG}$ ) and evaluated $12 \mathrm{~h}$ later for evidence of skin blisters. Following examination of the skin for blisters, animals were sacrificed. Clinical activity in mice was evaluated [Nikolsky's sign (Ns)] following the scale used elsewhere (16). Skin biopsies from each animal included lesional skin samples for conventional histological examination by haematoxylin/eosin (H\&E) and immunohistochemical (IHC) staining and IF techniques. Histological examination was evaluated following the scale described elsewhere (16). The present study was approved by the Ethics Committee of the University Clinic of Navarra, University of Navarra (02408). 


\section{Pretreatment of mice with inhibitors}

Inhibitors used in our study were administered by a single intradermal injection into the back of mice $2 \mathrm{~h}$ before PV-IgG administration, as described elsewhere $(15,16)$. All inhibitors were purchased from Calbiochem (Darmstadt, Germany). Firstly, each inhibitor was dissolved in DMSO and later diluted in PBS before injection into mice ( $n=3$ for each inhibitor) at the following doses, based on in vivo studies reported elsewhere (16) showing that the inhibitors used are specific for their targets and without any side effects in the mice: mTOR activity inhibitor rapamycin (RP), $5 \mu \mathrm{g} / \mathrm{g}$ body weight (33); Src activity inhibitor PP1, $1 \mu \mathrm{g} / \mathrm{g}$ body weight (32); pan-caspase inhibitor (cpm-VAD-CHO) (PCI), $1.6 \mu \mathrm{g} / \mathrm{g}$ body weight (34). Doses of both pan-HER isoform inhibitor CL-387,785 (CL) $(10 \mu \mathrm{g} / \mathrm{g}$ body weight) and FI PF-573,228 (FI of tyrosine phosphorylation in Y397) (dose $5 \mu \mathrm{g} / \mathrm{g}$ body weight) were based on the specificity studies of Sweeney et al. (31) and Slack-Davis et al. (35), respectively, and our studies of effectiveness, morbidity and mortality on mice. In preliminary studies, we excluded the effects of dermal manipulation and DMSO by injecting PBS or DMSO diluted in PBS. Mice were sacrificed $12 \mathrm{~h}$ after PV-IgG fractions injected intradermally.

\section{IHC staining procedure}

Skin samples from mice pretreated or not with inhibitors and injected with PV-IgG or NHS-IgG were obtained for IHC staining as described elsewhere $(15,16)$. A tissue microarray was constructed from each specimen in the cohort. Tissue cores of $2 \mathrm{~mm}$ in size were obtained from paraffin-embedded tissue blocks. Tissue sections were incubated with different polyclonal antibodies specific for P-mTOR (Ser2448), diluted to 1:50, citrate (Cell Signaling Technology, Beverly, MA, USA); P-Src (Tyr416), diluted to 1:100, EDTA (Cell Signaling Technology); FAK Y397, diluted to 1:100, Tris (Abcam, Cambridge, UK); FAK Y925, diluted to 1:100, Tris (Bioworld, Minneapolis, MN, USA). Negative controls were performed by omission of the primary antibodies. Sections were incubated overnight at $4{ }^{\circ} \mathrm{C}$ with the primary antisera. The ratio of stained cells on the basal layer (area of stained basal keratinocytes/area of total basal keratinocytes) was calculated using image analysis software on images acquired with a motorized Axioplan 2ie microscope (Zeiss, Oberkochen, Germany), operated through Meta-Morph imaging software (Molecular Devices, Downing-town, PA, USA), and fitted with a Zeiss Axio Cam ICc3 camera connected to a PC equipped with image-capturing software. Image analysis was performed using Matlab Software (The MathWorks Inc, Natick, MA, USA). The segmentation algorithm was custom-designed by Dr Ortiz-de-Solorzano's Morphology and Imaging Laboratory (CIMA, University of Navarra) (16).

\section{Measurement of caspase- 3 and caspase- 9 specific activities}

Caspase- 3 and caspase-9 activities were measured in skin biopsies from each animal using the Caspase-3 Activity Assay Kit Colorimetric (Sigma-Aldrich, St. Louis, MO, USA) and Caspase-9 Activity Assay Kit Colorimetric (Calbiochem, Darmstadt, Germany). When indicated, animals were pretreated with inhibitors in the conditions described above. Skin biopsies were resuspended in lysis buffer (buffer caspase-3 and caspase-9), and caspase-3 and caspase-9 activities were measured following the manufacturer's instructions. The value obtained from the analysis of the NHS samples was scored as 1. 


\section{Preparation of cell lysates and Western blot analysis}

Mouse skin proteins were extracted by homogenization of skin biopsy lysates obtained by sonication. Western blot analysis of protein samples was carried out after SDSPAGE gels (38) and transferred onto nitrocellulose membranes (GE Healthcare, Buckinghamshire, UK). Blots were incubated overnight at $4^{\circ} \mathrm{C}$ with different polyclonal rabbit antibodies, such as anti-Bax and antiBcl-2 (Cell Signaling Technology), or monoclonal antibodies, such as anti- $\beta$-actin (Sigma-Aldrich). Antibodies were diluted according to the manufacturer's instructions. Membranes were washed with T-TBS and incubated for $40 \mathrm{~min}$ at room temperature with the appropriate secondary antibody diluted 1:10000. After washing, blots were developed using the ECL system Super Signal ULTRA kit (Thermo Scientific, Rockford, IL, USA), and immunoreactive proteins were visualized on High performance chemiluminescence film Amersham (Buckinghamshire, England) Hyperfilm ${ }^{\mathrm{Tm}}$ ECL. Densitometric analyses were carried out using a GS-800 calibrated densitometer (BioRad, Hercules, CA, USA). When necessary, striping treatment was carried out. Equivalent loading was confirmed using an antibody against actin.

\section{Statistical analysis}

All results are expressed as mean $\pm \mathrm{SD}$. Data were analysed using the nonparametric Kruskal-Wallis test to determine the differences between all independent groups. When significant differences were obtained $(\mathrm{P}<0.05)$, differences between two groups were tested using the Mann-Whitney U test.

\section{RESULTS}

Mice injected with PV-IgG show increased levels of FAK phosphorylated on tyrosine residues 397 and 925

Using our animal model, normal human serum (NHS)-IgG injected into mice did not induce PV lesions (Fig. 1), whereas injection of PV-IgG reproduced clinical PV lesions, with the presence of suprabasal acantholysis (Fig. 1). This clearly demonstrates that our mouse model was appropriate for investigating the mechanisms implicated in PV.

Firstly, we studied the presence of P-FAK (Y397/925) in the basal layer of the epidermis. P-FAK on both Y397/925 residues showed a plasma membrane pattern in the basal cells of the epidermis in mice injected with NHS-IgG (Figure S1a-c). Also, after PV-IgG administration, the expression of P-FAK in these two tyrosine residues (397/925) revealed an uniform staining throughout the cytoplasm of the basal cells of the epidermis only in acantholytic areas, with a higher and significant increased level to that found after NHS-IgG administration $(\mathrm{P}<0.001)$ (Figure S1a-c). Nonacantholytic/perilesional areas only showed a peripheral membrane pattern (data not shown).

\section{Suprabasal acantholysis induced by PV-IgG was decreased if mice were pretreated with FI}

The next step was to determine whether FAK phosphorylation on tyrosine residues 397 and 925 mighty contribute to the development of PV lesions in our mouse model. 
In other words, we wanted to know whether FAK is only a passive bystander or on the contrary it plays a key role in the detachment of cells in PV. Therefore, we administered an inhibitor of FAK activity prior to PV-IgG injection to mice and compared the results with those observed when mice were injected either with NHSIgG or with PV-IgG. None of the mice pretreated with the FI exhibited either clinical or histological findings of PV (Fig. 1). Therefore, our results clearly strengthen the hypothesis of a highly significant role of FAK activity in the detachment process observed in PV lesions.

Pretreatment with inhibitors of $\mathrm{mTOR}$, Src, HER isoforms and pan-caspases along with PV-IgG administration in mice may decrease the expression of FAK phosphorylated on Y397 and Y925 residues

After revealing a novel biochemical mechanism for FAK in PV, our next step was to understand the underlying mechanism. We decided to investigate whether a relationship could be established between FAK, and HER isoforms, Src, mTOR and caspases. To answer this question, we administered inhibitors for each of these molecules before PV-IgG treatment. P-FAK on tyrosine residues 397 and 925 was statistically lower if mice were pretreated with HER isoforms $(\mathrm{P}<0.001)$, Src $(\mathrm{P}<$ 0.001 and $\mathrm{P}<0.01$, respectively), mTOR ( $\mathrm{P}<0.001$ and $\mathrm{P}<0.01$, respectively) or PCIs $(\mathrm{P}<0.001)$ followed by PV-IgG injection (Figure S1a-c). In addition, P-FAK (Y397/925) presented a plasma membrane pattern in the basal cells of the epidermis, exactly the same as that observed after NHS-IgG injection and completely opposite to that observed after the injection of PV-IgG (Figure S1a). Also, clinical PV lesions (Nikolsky's sign) and suprabasal acantholysis in mice were absent when these inhibitors were used (data not shown).

Inhibition of suprabasal acantholysis of mice pretreated with FI is associated with a decrease in the changes in both Bax and Bcl-2 levels, and caspase-9 and caspase-3 activities induced by PV-IgG

After establishing a key role of P-FAK in PV lesions, we wondered how this might occur and what mechanisms were acting in this process. Skin samples from mice pretreated with FI plus PV-IgG were obtained to test whether this inhibitor might affect Bax and Bcl-2 levels measured by western blot assay. The results were compared with those obtained in skin samples taken from mice injected only with PVIgG or NHS-IgG. When PV-IgG were injected into mice, Bcl-2 and Bax levels were significantly lower and higher, respectively, compared with those found in mice pretreated with FI $(\mathrm{P}<0.001)$ (Fig. 2a,b). Similar results were found when mice were pretreated with HER isoforms $(\mathrm{P}<0.001$ and $\mathrm{P}<0.01$, respectively), mTOR $(\mathrm{P}<$ 0.001 and $\mathrm{P}<0.01$, respectively) and $\operatorname{Src}$ inhibitors $(\mathrm{P}<0.001)$ (Fig. 2a,b). Subsequently, we decided to investigate whether FI also regulated caspase-3 and caspase-9 activities using similar skin samples. Interestingly, we found that the significant increase in caspase- 3 and caspase- 9 activities after PV-IgG injection was totally absent when mice were pretreated with the FI $(\mathrm{P}<0.001)$. Identical results were obtained when mice were pretreated with HER isoforms $(\mathrm{P}<0.001)$, mTOR $(\mathrm{P}<$ $0.001)$ or Src $(\mathrm{P}<0.001)$ inhibitors before PV-IgG administration (Fig. 2c). All these findings clearly show that FAK, the same as HER isoforms, mTOR and Src (16), may contribute to acantholysis in PV lesions by affecting Bcl-2/Bax ratio and caspase-3 and caspase- 9 activities. 


\section{FI pretreatment reduced the increase in phosphorylated Src and mTOR expression induced by PV-IgG in the basal layer of the epidermis}

In the present study, we wondered whether FAK may regulate Src and mTOR in PV. Expression levels of P-Src and P-mTOR in the basal layer of the epidermis were significantly higher after PV-IgG injection, when compared with those obtained after NHS-IgG administration ( $\mathrm{P}<0.01$ and $\mathrm{P}<0.001$, respectively) (Fig. 3a,b). Interestingly, the expression of phosphorylated forms of both kinases was statistically lower when mice were pretreated with the FI before PV-IgG administration $(\mathrm{P}<$ 0.001) (Fig. 3a,b). Therefore, FAK may regulate and be regulated by these signalling molecules (Src and mTOR) and contribute to orchestrate the acantholysis observed in PV lesions.

\section{DISCUSSION}

Many relevant findings have established that the mechanisms involved in the blistering formation in PV are the result of many intracellular molecular events articulated in conjunction (multiplehit theory) (6). Thus, several molecules and intracellular signalling pathways play an crucial role in the detachment of keratinocytes in PV lesions: the phosphatidylinositol-4,5-diphosphate pathway (11), p38 MAP kinase (14), nNOS (15), Rho protein (39), HER isoforms (16,21), mTOR (16) and Src kinases $(5,12,16)$.

Several findings have been taken into account before exploring the role of FAK in the development of PV lesions: the FAK protein has been found to be increased in acantholytic cells in PV (29); also, antibodies against AChR in patients with PV (4) upregulate the degree of phosphorylation of FAK (13); FAK plays a role in many cellular functions via a cross-talking mechanism with integrins and HER isoforms (40); and an important effect of FAK on cytoskeleton may be mediated by mTOR kinase activation (31), which plays a role in PV lesions (16).

Usually, FAK is activated by phosphorylation on different tyrosine residues, either through autophosphorylation or through phosphorylation by other kinases such as Src kinases (41). Phosphorylated FAK on Y397 interacts with SH2-containing proteins including Src, Shc, phosphatidylinositol 3-kinase, growth factor receptor-bound protein 7 and phospholipase C (28). This is followed by Src-mediated phosphorylation and activation of FAK on residues Y576/577 in the kinase domain activation loop (42). Src can also phosphorylate FAK on Y407, Y861 and Y925 (43). Our study describes for the first time that activated P-FAK (Y397/925) plays an important role in the detachment of cells in PV lesions. P-FAK expression showed a plasma membrane pattern in the basal cells of the epidermis after NHS-IgG administration to mice, such as described in the epidermis of non-treated mice (27). However, when mice were injected with PV-IgG, P-FAK (Y397/925) levels increased showing a diffuse cytoplasmic pattern in the basal cells of epidermis. This raises the question of which mechanisms are involved in these changes.

One of the most relevant findings in this study was the inhibition of suprabasal acantholysis by pretreatment of PV-IgG injected mice with FI. Caspase activation and an increase in the BaxBcl-2 ratio also seem to be involved in PV development (17-22) as suggested in our study. Recently, reorganization of cortical actin filaments has been found to be a critical step in PV-IgG-induced keratinocyte dissociation (44). Usually, detachment of keratinocytes in PV occurs by the condensation of cytoskeletal 
filaments even before the separation of desmosomes is observed on the lateral sides of the basal layer of epidermis (45). mTOR may participate in the development of PV lesions (16) by affecting Bcl-2 phosphorylation/degradation as it has been described in damaged microtubule cells (46). Recently, FAK has been implicated in the constellation of pathways that regulate cytoskeletal dynamics $(47,48)$, in a process mediated by mTOR upregulation (36). Therefore, our previous observation of the abolition of acantholysis induced by PV-IgG in mice pretreated with rapamycin (16), along with the present results showing a diminished effect of PVIgG on both the Bax/Bcl-2 ratio and caspase- 3 and caspase-9 activities in the presence of mTOR or FIs, strongly supports the role of FAK in acantholysis in PV in our mouse model. This hypothesis is supported by the findings of Ravikumar et al. (49), showing that rapamycin inhibited apoptosis in a cytochrome c and caspase-3- and caspase-9dependent process.

An important and unresolved issue is to determine whether caspase activation contributes to PV development in either an apoptosis-dependent or apoptosisindependent form. Usually, caspase activation results in the cleavage of several substrates implicated in different aspects of apoptosis such as nuclear fragmentation and cytoskeletal rearrangement (50). However, other additional roles different from apoptosis have been identified for these proteases $(51,52)$. Wang et al. demonstrated that caspase-3 and caspase-1 were activated several hours before cell detachment induced by PV-IgG in keratinocyte culture (19). Frusic-Zlotkin et al. obtained similar results measuring caspase- 8 and caspase-3 (25). Also, our present and previous results and several other reports confirm that pan-caspase inhibitor may abolish PV and pemphigus foliaceus acantholysis (16,53). Recently, Cirillo et al. (54) have observed that caspases may be activated as an early pathogenic event in PV acantholysis, whereas apoptosis is only a late finding not necessary for acantholysis. These authors conclude that although apoptosis may be observed in PV, the pathogenic event leading to acantholysis is the activation of caspases. Several research groups have reported a direct effect of caspases on FAK mainly caspase-3, caspase- 6 and caspase-7 and a cleavage of this kinase during apoptosis $(55,56)$. In the present study, we have shown that during PV development, the location of FAK in the cell is modified by caspases probably due to the fragmentation of itself and other cytoskeletal proteins. On the other hand, the blocking effect of pan-caspase inhibitor on PV-IgG induced FAK phosphorylation seems to indicate the existence of a positive loop that would contribute to the progression of the acantholytic process (Fig. 4). This positive loop would be generated by the activation of some protein kinase by caspases (Fig. 4) such as has been described in other systems (57). One of the kinases activated by caspases is MEKK1, which is involved in the activation of p38, an apoptotic kinase that participates in PV acantholysis (14). In this sense, activation of caspases might contribute to the disruption of the desmosome and disintegration of the intermediate filament system $(58,59)$.

Another relevant issue is the mechanism implicated in FAK activation in PV lesions and its relationship with other kinases. Our results indicate a relationship between HER isoforms, Src kinase and FAK, showing a significant decreased expression of PFAK (Y397/925) in PV-IgG-injected mice by pretreatment with Src and HER inhibitors. These findings may be explained taking into account that FAK may be upregulated by recruitment and interaction through its unique FAK N-terminal domain with P-EGFR (40), which is increased in mice treated with PV-IgG (16). In addition, upon the stimulation of EGFR, recruited FAK is auto- or transphosphorylated at Y397 generating a docking site for Src. Because phosphorylation by Src represents a relevant 
step to induce the activation of FAK, as has been reported above $(28,41)$, Src inhibitor PP1 may help to abolish acantholysis, at least in part, by the inhibition of FAK activation. Therefore, our previous findings showing the absence of clinical and histological lesions in mice pretreated with PP1 and injected with PV-IgG $(12,16)$ may be explained, at least in part, by the inhibition of Src-mediated activation of FAK (Fig. 4). Indeed, the effect of FAK on Src phosphorylation degree could be explained by the capacity of P-FAK (Y397) to recruit and activate Src (28).

Some authors suggest that mTOR might switch its kinase activity to transmit survival or death signals (60). Also, it has been described that inhibition of PI3K/Akt signalling increases death signals initiated by damaged microtubules leading to the phosphorylation/degradation of Bcl-2 by mTOR (46). In this way, the absence of Akt in the basal cells of the epidermis (61) would increase the amount of mTOR that may be phosphorylated and activated by Src (62) leading to Bcl-2 phosphorylation/degradation and subsequent caspase activation in mice injected with PV-IgG.

In conclusion, in PV acantholysis, FAK, Src and mTOR seem to be activated as a result of HER isoform stimulation in a molecular platform possibly generated by adapter and scaffold proteins that provide multiple docking sites for an array of signalling and structural proteins. Some of the proteins in this platform may receive information from Dsg or non-Dsg autoantigen receptors in PV $(4,5)$ and hence modulate the acantholytic response. As this study has been carried out in a mouse model, future investigations using skin/mucosa from the patients with PV are needed in order to analyse the role of these signalling molecules in human PV. However, the successful use of mTOR inhibitors in patients with PV $(63,64)$ underlines the relevance of our findings. Indeed, better insight into the signalling cascades involved in PV acantholysis might expand the number of therapeutic options for this blistering disease.

\section{ACKNOWLEDGEMENTS}

We thank Professor Esteban Santiago for his kind contribution to the final version of this manuscript. This work was supported by a FIS grant from the Ministry of Health of Spain (PI081591). Dr. Modol and Dr. Gil performed the research, Dr. LópezZabalza and Dr. España designed the research study, all authors analysed the data, and Dr. López-Zabalza and Dr. España wrote the paper.

\section{CONFLICT OF INTEREST}

The authors have declared no conflicting interests.

\section{REFERENCES}

1. Stanley J R. J Clin Invest 1989: 83: 1443-1448.

2. Ding X, Aoki V, Mascaro J M Jr etal. J Invest Dermatol 1997: 109: 592-596.

3. Muller R, Svoboda V, Wenzel E etal. Exp Dermatol 2007: 17: 35-43.

4. Nguyen V T, Ndoyen A, Shultz L D etal. J Clin Invest 2000: 106: 1467-1479. 
5. Chernyavsky A I, Arredondo J, Kitajima Y et al. J Biol Chem 2007: 282: 13804-13812.

6. Grando S A. Dermatology 2000: 201: 290-295.

7. Evangelista F, Dasher D A, Diaz L A etal. J Invest Dermatol 2008: 128: 1710_ 1718.

8. Marchenko S, Chernyavsky A I, Arredondo J et al. J Biol Chem 2010: 285: 3695-3704.

9. Sitaru C, Zillikens D. Exp Dermatol 2005: 14: 861-875.

10. Esaki C, Seishima M, Yamada T etal. J Invest Dermatol 1995: 105: 329-333.

11. Seishima M, Esaki C, Osada K etal. J Invest Dermatol 1995: 104: 33-37.

12. Sánchez-Carpintero I, España A, Pelacho B etal. Br J Dermatol 2004: 151: 565-570.

13. Chernyavsky A I, Arredondo J, Vetter D E etal. Exp Cell Res 2007: 313: 35423555.

14. Berkowitz P, Hu P, Liu Z etal. J Biol Chem 2005: 280: 23778-23784.

15. Marquina M, España A, Fernández-Galar M et al. Br J Dermatol 2008: 159: 68-76.

16. Pretel M, España A, Marquina M etal. Exp Dermatol 2009: 18: 771-780.

17. Puviani M, Marconi A, Cozzani E etal. J Invest Dermatol 2003: 120: 164-167.

18. Pelacho B, Natal C, España A etal. FEBS Lett 2004: 566: 6-10.

19. Wang X, Bregegere F, Frusic-Zlotkin M etal. Apoptosis 2004: 9: 131-143.

20. Frusic-Zlotkin M, Pergamentz R, Michel B etal. Ann N Y Acad Sci 2005: 1050: 371-379.

21. Frusic-Zlotkin M, Raichenberg D, Wang X etal. Autoimmunity 2006: 39: $563-$ 575.

22. Bektas M, Jolly P, Rubenstein D S. Dermatol Res Pract 2010: 2010: 456841.

23. Grando S A, Bystryn J C, Chernyavsky A I etal. Exp Dermatol 2009: 18: 764 770.

24. Schmidt E, Waschke J. Autoimmun Rev 2009: 8: 533-537.

25. Craig S W, Johnson R P. Curr Opin Cell Biol 1996: 8: 74-85.

26. Tomar A, Schlaepfer D D. Curr Opin Cell Biol 2009: 21: 676-683.

27. Essayem S, Kovacic-Milivojevic B, Baumbusch C et al. Oncogene 2006: 25: 1081-1089.

28. Li S, Hua Z-C. Adv Cancer Res 2008: 101: 45- 61.

29. Penneys N S. Br J Dermatol 1996: 135: 592-594.

30. Nguyen V T, Ndoye A, Grando S A. Am J Pathol 2000: 157: 1377-1391.

31. Sweeney W E, Chen Y, Nakanishi K etal. Kidney Int 2000: 57: 33-40.

32. Jadhav V, Matchett G, Hsu F P et al. J Neurosurg 2007: 106: 680-686.

33. Teachey D T, Obzut D A, Axsom K etal. Blood 2006: 108: 1965-1971.

34. Imao M, Nagaki M, Imose M etal. Liver Int 2006: 26: 137-146.

35. Slack-Davis J K, Martin K H, Tilghman R E et al. J Biol Chem 2007: 282: 14845-14852.

36. Liu L, Chen L, Chung J etal. Oncogene 2008: 27: 4998-5010.

37. Amagai M, Tsunoda K, Zillikens D etal. J Am Acad Dermatol 1999: 40: 167170.

38. Laemmli U K. Nature 1970: 227: 680-685.

39. Waschke J, Spindler V, Bruggeman P etal. J Cell Biol 2006: 175: 721-727.

40. Sieg D J, Hauck C R, Ilic D etal. Nat Cell Biol 2000: 2: 249-257.

41. Hanks S K, Ryzhova L, Shin N Y etal. Front Biosci 2003: 8: d982-d996.

42. Owen J D, Ruest P J, Fry D W et al. Mol Cell Biol 1999: 19: 4806-4818. 
43. Cary L A, Guan J L. Front Biosci 1999: 4: d102-d113.

44. Gliem M, Heupel W M, Spindler V etal. Am J Physiol Cell Physiol 2010: 299: C606-C613.

45. Shimizu A, Ishiko A, Ota T etal. J Invest Dermatol 2004: 122: 1145-1153.

46. Asnaghi L, Calastretti A, Bevilacqua A etal. Oncogene 2004: 23: 5781-5791.

47. Schober M, Raghavan S, Nikolova M etal. J Cell Biol 2007: 176: 667-680.

48. Broussard J A, Webb D J, Kaverina I. Curr Opin Cell Biol 2008: 20: 85-90.

49. Ravikumar B, Berger Z, Vacher C et al. Hum Mol Genet 2007: 15: 1209-1216.

50. Thornberry N A, Lazebnik Y. Science 1998: 281: 1312-1316.

51. Ghayur T, Banerjee S, Hugunin M etal. Nature 1997: 386: 619-623.

52. Lüthi A U, Martin S J. Cell Death Differ 2007: 14: 641-650.

53. Lin N, Zhao M, Wang J etal. J Immunol 2009: 182: 711-717.

54. Cirillo N, Lanza A, Prime S. Apoptosis-independent caspase activation as an early pathogenic event in the autoimmune skin disease pemphigus vulgaris. Poster presented in JC Bystryn pemphigus \& pemphigoid meeting: from the bench to the bedside. Bethesda, ML, USA, November 5-6, 2010.

55. Wen L P, Fahrni J A, Troie S etal. J Biol Chem 1997: 272: 26056-26061.

56. Mian M F, Kang C, Lee S etal. Chem Biol Interact 2008: 171: 57-66.

57. Natal C, Oses-Prieto J A, Pelacho B etal. Apoptosis 2006: 11: 209-219.

58. Weiske J, Schöneberg T, Schro“dr W etal. J Biol Chem 2001: 276: 4117541181.

59. Dusek R L, Getsios S, Chen F etal. J Biol Chem 2006: 281: 3614-3624.

60. Asnaghi L, Bruno P, Priulla M etal. Pharmacol Res 2004: 50: 545-549.

61. Calauti E, Li J, Saoncella S etal. J Biol Chem 2005: 280: 325856-325865.

62. Vojtehova M, Turrerkova J, Kucerova D etal. Neoplasia 2008: 10: 99-107.

63. Saggar S, Zeichner J A, Brown T T etal. Arch Dermatol 2008: 144: 654-657.

64. Grando S A, Laquer V T, Le H M. J Am Acad Dermatol 2011: 65: 684-686. 


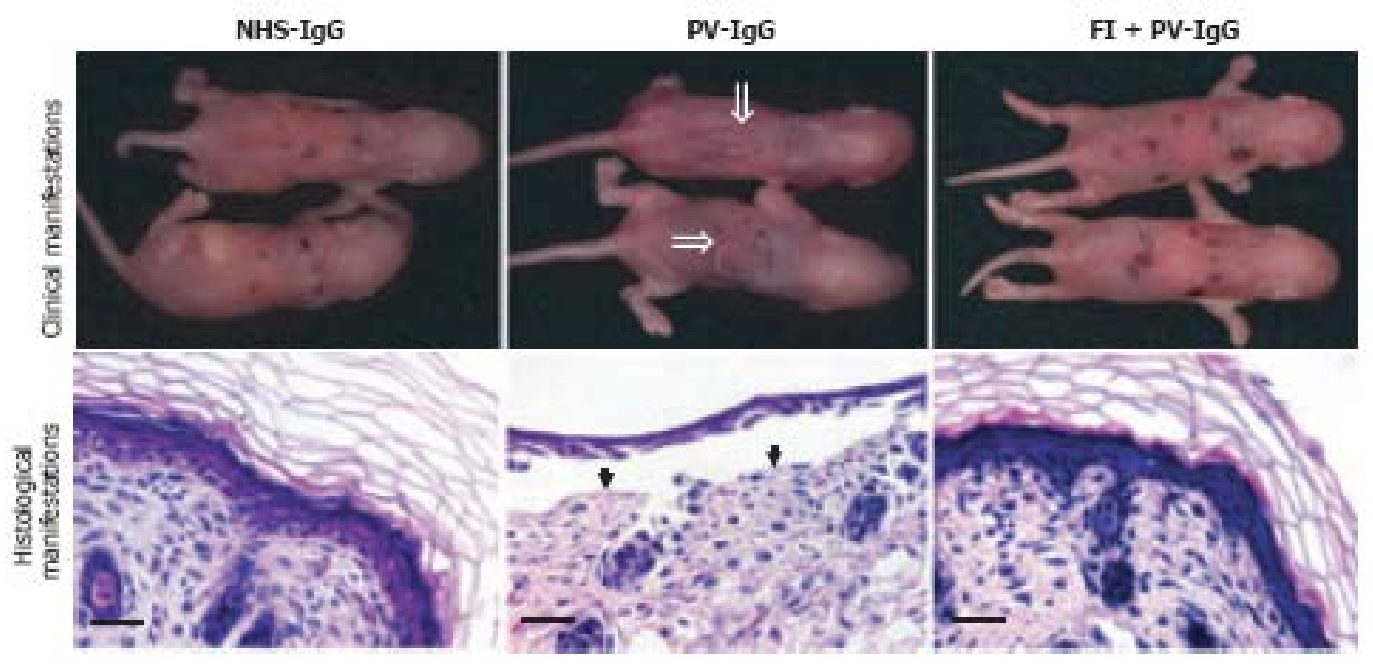

Figure 1. Clinical and histological findings after NHS-IgG, PV-IgG, or FAK inhibitor (FI) plus PV-IgG injections. Mice were injected on the back (dotted area of skin) with NHS-IgG or PV-IgG. When NHS-IgG were administrated to mice, no clinical or histological findings (acantholysis) of PV were found. However, if mice were treated with PV-IgG, detachment of skin (Nikolsky's sign $\Downarrow$ ) was observed and suprabasal acantholysis was detected $(\downarrow)$. Those mice pretreated with FI prior to PVIgG injection did not show either clinical or histological PV lesions. FAK, focal adhesion kinase; NHS, normal human serum; PV, pemphigus vulgaris; (1), PV-IgG versus NHS-IgG; (2), FI plus PV-IgG versus PV-IgG; bars = $50 \mathrm{~lm}$. 
(a)

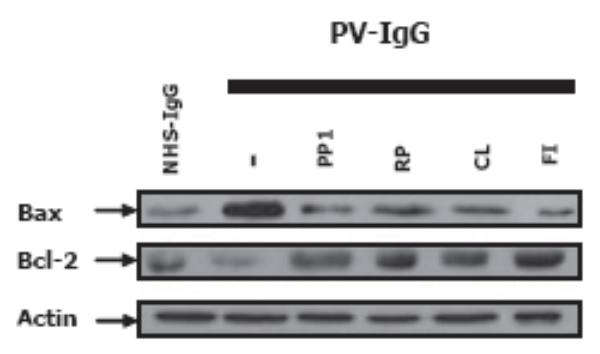

(b)

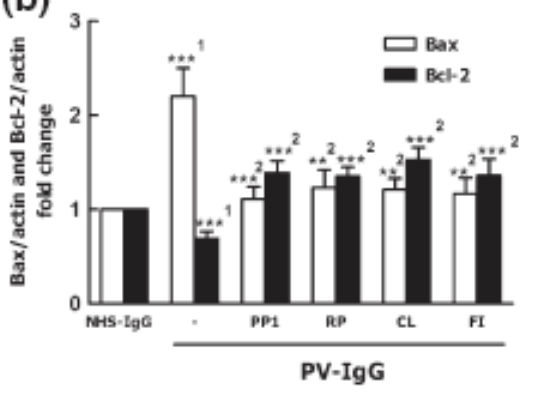

$\square$ Caspase-3

- Caspase-9

(c)

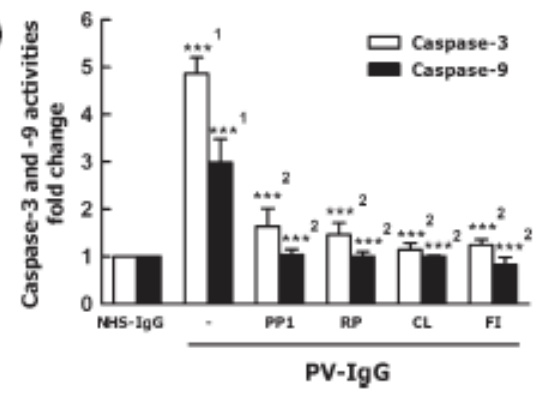

Figure 2. Mice injected with PV-IgG showed higher and lower levels of Bax and Bcl2 respectively, and higher caspase- 3 and caspase- 9 activities, compared with mice injected with either NHS-IgG or mice pretreated with inhibitors before PV-IgG administration. (a) Levels of Bax and Bcl-2 analysed by western blotting, (b) quantitation of blots and (c) quantitation of caspase-3 and caspase-9 activities, when mice were treated with NHS-IgG, and PV-IgG only or pretreated with inhibitors. NHS, normal human serum; PV, pemphigus vulgaris; HER isoforms (CL), Src (PP1), mTOR (RP), FAK (FI) and pan-caspases (PCI) inhibitors; (1), PV-IgG versus NHS-IgG; (2), several inhibitors plus PV-IgG versus PV-IgG; **P $<0.01$; ***P $<0.001$; bars $=50$ $\mathrm{lm}$. The results are expressed as mean $\pm \mathrm{SD}$ of nine independent experiments. 

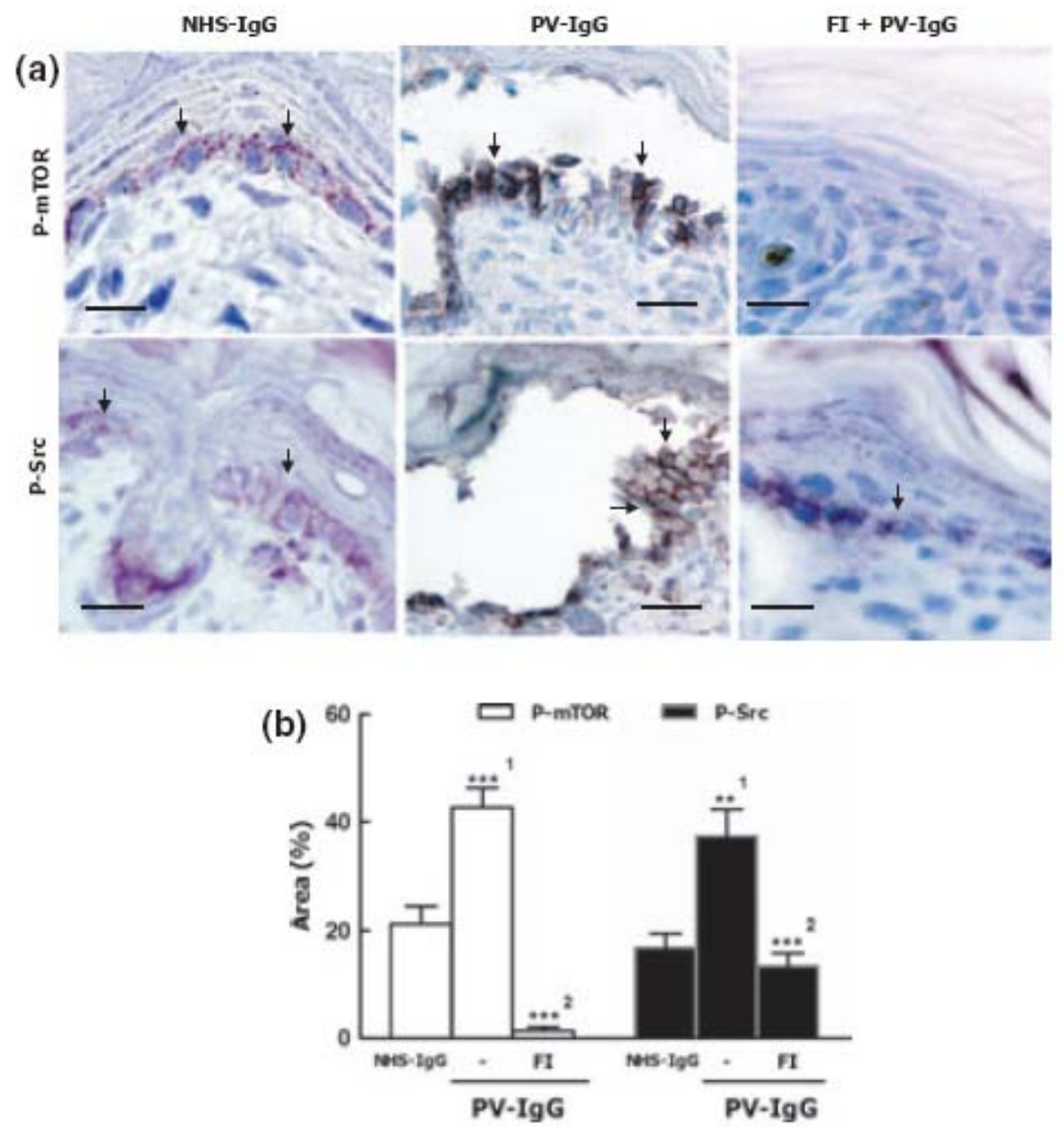

Figure 3. Mice injected with PV-IgG showed a higher expression of both P-Src and PmTOR kinases in the basal cells of the epidermis when compared with those injected with either NHS-IgG or pretreated with focal adhesion kinase (FAK) inhibitor before PV-IgG injection. (a) P-Src and P-mTOR immunohistochemistry detection after NHSIgG, PV-IgG or FI plus PV-IgG administration ( $\downarrow$ ) and (b) quantitation of immunohistochemistry. FI, FAK inhibitor; PV, pemphigus vulgaris; NHS, normal human serum; P, phosphorylated; mTOR, mammalian target of rapamycin; (1), PVIgG versus NHS-IgG; (2), several inhibitors plus PV-IgG versus PV-IgG; **P $<0.01$; $* * * \mathrm{P}<0.001$; bars $=50 \mathrm{~lm}$. The results are expressed as mean $\pm \mathrm{SD}$ of nine independent experiments. 


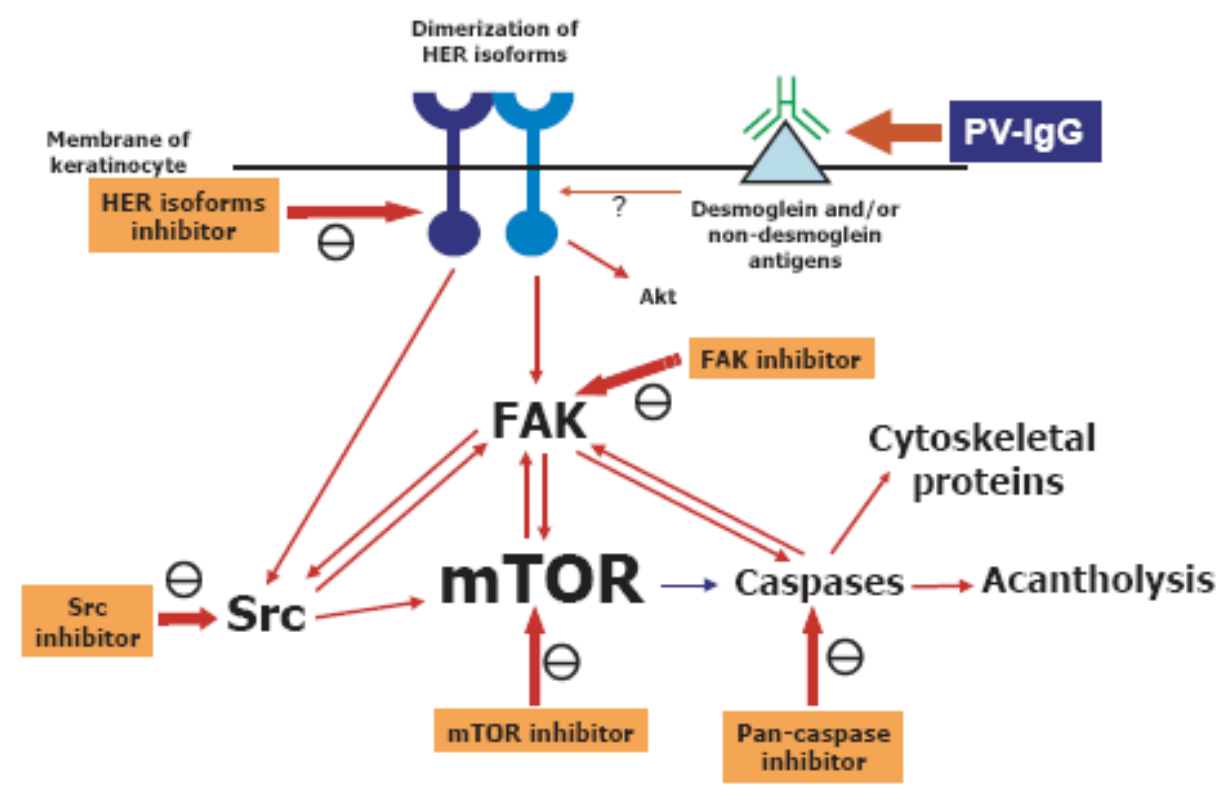

Figure 4. Hypothetical connection between HER isoforms, focal adhesion kinase (FAK), Src, mammalian target of rapamycin (mTOR) and caspases during pemphigus vulgaris (PV) acantholysis in the basal layer of the epidermis. Our present and previous findings (16) indicate that FAK, Src and mTOR may be activated as a result of HER isoforms' stimulation. In addition, mTOR may increase caspase activity by inducing phosphorylation / degradation of Bcl-2. In turn, a positive loop might be generated by caspases acting on FAK and other kinases, and cytoskeletal proteins in order to promote the progression of the acantholytic process. Mutual effects between FAK and Src, mTOR or caspases would also contribute to achieve acantholysis in PV lesions. 